\title{
Single versus double row suture anchor fixation for greater tuberosity fractures - a biomechanical study
}

\author{
Gernot Seppel ${ }^{1,2^{*}+}$, Tim Saier ${ }^{1,3 \dagger}$, Frank Martetschläger ${ }^{1,4}$, Johannes E. Plath ${ }^{1,5}$, Alberto Guevara-Alvarez ${ }^{1}$, \\ Julia Henschel ${ }^{6}$, Martin Winkler ${ }^{6}$, Peter Augat ${ }^{6,7}$, Andreas B. Imhoff ${ }^{1}$ and Stefan Buchmann ${ }^{1,8}$
}

\begin{abstract}
Background: Fractures of the humeral greater tuberosity (GT) are a frequent injury progressively treated with arthroscopic suture anchor repair. Yet, no biomechanical study has been performed comparing fixation strength of arthroscopic single- (SR) vs. double row (DR) fixation.

Methods: Standardized fractures of the greater tuberosity were created in 12 fresh frozen proximal humeri. After random assignation to the SR or DR group the fixed humeri were tested applying cyclic loading to the supraspinatus and infraspinatus tendon. Load to failure and fragment displacement were assessed by means of an electrodynamic material testing machine using an optical tracking system.

Results: Load to failure values were higher in the DR group (649 N; \pm 176$)$ than in the SR group (490 N; \pm 145$)$ however without statistical significance $(p=.12)$. In greater tuberosity displacement of 3-5 mm surgical treatment is recommended. The fixing constructs in this study did not reach displacement landmarks of 3 or $5 \mathrm{~mm}$ before construct failure as shown in previous studies. Thus the applied traction force $(\mathrm{N})$ at $1 \mathrm{~mm}$ displacement was analyzed. In the SR group the load at $1 \mathrm{~mm}$ displacement was $277 \mathrm{~N} ; \pm 46$ compared to $260 \mathrm{~N} ; \pm 62$ in the DR group $(p=.65)$.

Conclusion: The results suggest that both techniques are viable options for refixation of greater tuberosity fractures.

Level of Evidence: Laboratory study.
\end{abstract}

Keywords: Greater tuberosity fracture, Suture anchor, Arthroscopic, Double row fixation, Single-row

\section{Background}

Isolated fractures of the greater tuberosity represent a common injury accounting for up to $1 / 5$ th of all proximal humeral fractures $[1,10]$. Due to the limited dimension of the subacromial space even small residual superior displacement may cause clinical impairment [2,9]. It has been shown, that conservative treatment for dislocated greater tuberosity fractures is associated with pain and impaired range of motion (ROM) [8]. Therefore, especially

\footnotetext{
* Correspondence: seppel@tum.de

${ }^{\dagger}$ Equal contributors

${ }^{1}$ Department of Orthopedic Sports Medicine, Klinikum rechts der Isar, Technical University Munich, Ismaninger Str. 22, 81675 Munich, Germany ${ }^{2}$ Department of Orthopedics and Trauma Surgery, Krankenhaus Barmherzige Brüder, Munich, Germany

Full list of author information is available at the end of the article
}

in patients with high functional demands surgical treatment is recommended if superior dislocation of the greater tuberosity reaches 3-5 $\mathrm{mm}$ of displacement $[4,9]$. Gerber and Warner described that malunion is one of the most common complications following surgical treatment of proximal humeral fractures [9]. As a general rule, it is well known that bony healing depends critically on anatomical reposition and rigid retention [31]. Besides, mechanical stability is essential to allow early functional rehabilitation in order to achieve satisfying clinical results [15]. Arthroscopic treatment of greater tuberosity fractures is an established method [12, 28, 29]. Previous biomechanical studies demonstrated a secure retention and reposition using suture anchors [3]. However, none of theses studies investigated on the influence of the postero- 
superior rotator cuff (M. infraspinatus (ISP) and M. supraspinatus (SSP)) forces on the stability of greater tuberosity fractures that were treated arthroscopically using suture anchor techniques. Further, to the knowledge of the authors, there is no biomechanical data comparing single vs. double-row anchor fixation.

The aim of this biomechanical study was to compare knotless suture anchor reconstruction in greater tuberosity fractures using a single- vs. double-row fixation. Therefore, a biomechanical human cadaveric in vitro model was established, that incorporated forces of the postero-superior rotator cuff on the greater tuberosity.

The primary hypothesis of this study was, that doublerow fixation confers greater load-to-failure strength and less secondary displacement under cyclic loading compared to single-row fixation of the greater tuberosity.

\section{Material \& Methods}

This biomechanical study was approved by the institutional review board of the Technical University Munich, Munich, Germany.

\section{Specimen preparation}

Testing was performed using 6 paired (right vs. left) freshfrozen human cadaveric shoulder specimens (6 male cadavers; mean age, 61,3 years; age range, 45 to 68 years). Specimens were thawed at room temperature $24 \mathrm{~h}$ before testing. The humeri (with completely preserved $\mathrm{Mm}$. supraspinatus and infraspinatus) were dissected from the specimens after disarticulation and removal of all other soft tissues (Fig. 1).

For inclusion, several criteria were mandatory: (1) intact supero-posterior rotator-cuff; (2) BMD >.50 g/ $\mathrm{cm}^{2}$; (3) no history and/or signs of previous fracture, and (4) cadaveric age younger than 70 years.

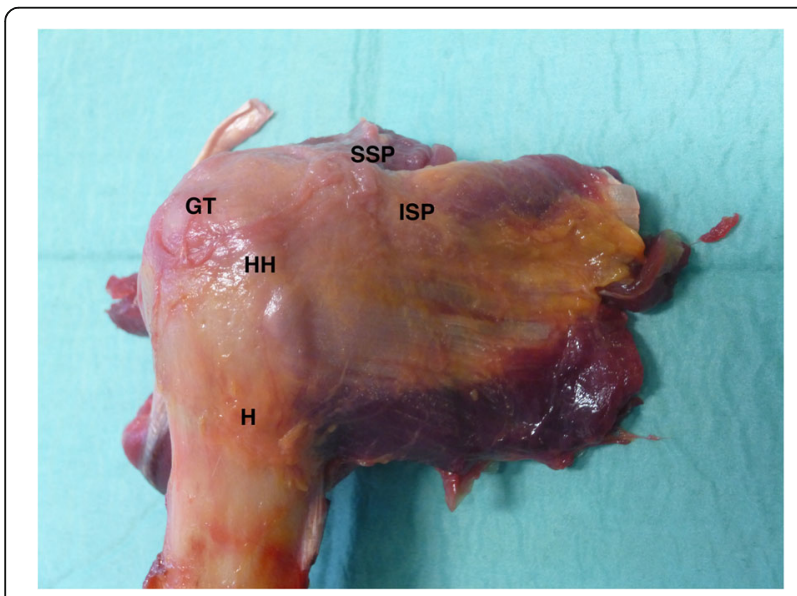

Fig. 1 Specimen; (H: Humerus; HH: Humeral Head; GT: Greater Tuberosity; SSP: Supraspinatus; ISP: Infraspinatus)
Bone mineral density (BMD) measurement (dual-energy $\mathrm{x}$-ray absorptiometry [in grams per square centimeter]) was performed for each specimen to guarantee consistent BMD values between groups (mean BMD, $0.65 \mathrm{~g} / \mathrm{cm}^{2}$; range, 0.58 to $0.67 \mathrm{~g} / \mathrm{cm}^{2}$ ).

The distal condyles were removed and the humeral shaft was adjusted to a standardized length of $20 \mathrm{~cm}$. A standardized greater tuberosity fracture was created in a $50^{\circ}$ degree angle to the humerus shaft using a chisel as described by Lin et al. [17].

\section{Biomechanical testing}

After anatomical reposition and temporary fixation using $2 \mathrm{~mm} \mathrm{~K}$-wires paired specimens (right vs. left) were randomly assigned to the following groups (each $n=6$ ) by lottery:

(1) Single-row suture tape (FiberTape, Arthrex Inc., Naples, USA) reconstruction using knotless anchor fixation with two anchors (PEEK SwiveLock 4,75 mm, Arthrex Inc., Naples, USA). Using Mason-Allen-stiches SSP-tendon was armed $1.5 \mathrm{~cm}$ from the anatomical footprint and the superior ISP-tendon $1 \mathrm{~cm}$ medial from the footprint with two suture tapes. In the tension direction of the SSP and according to the manufacturer instructions the two suture tape loaded anchors were placed laterally and $5 \mathrm{~mm}$ distally to the fracture into the humeral head (Fig. 2 a, b).

(2) Double-row suture tape (FiberTape, Arthrex Inc., Naples, USA) reconstruction using knotless anchor fixation with four anchors (PEEK SwiveLock 4,75 mm, Arthrex Inc., Naples, USA). First 2 suture tape loaded anchors were placed medial to the fracture at the cartilage-bone-transition-zone. At the identical anatomical sites as described above the tendons of SSP and ISP where perforated and the tapes where shuttled through the tendons, then the suture tapes were crossed. In the direction of movement for the SSP according to the manufacturer instructions two suture tape loaded anchors were placed laterally and $5 \mathrm{~mm}$ distally to the fracture into the humeral head. The anchors insertion sites were prepared by a $4 \mathrm{~mm}$ drill. (Fig. $3 \mathrm{a}$ and b)

All tests were performed at room temperature, and the surface of the specimens was constantly kept moist using isotonic saline solution.

To perform biomechanical testing, the humerus shaft of each specimen - adjusted to a standardized length of $20 \mathrm{~cm}$ - was potted and rigidly fixed in casting resin (RENCAST FC53; Huntsman Advanced Materials, Bergkamen, Germany) using a custom-made jig $[3,17]$. Optical markers were in line with the line of force and rigidly attached to the humeral head and greater tuberosity to measure relative movements between fragments using an optical tracking system (Pontos, GOM, Braunschweig, Germany). This system recorded displacement 

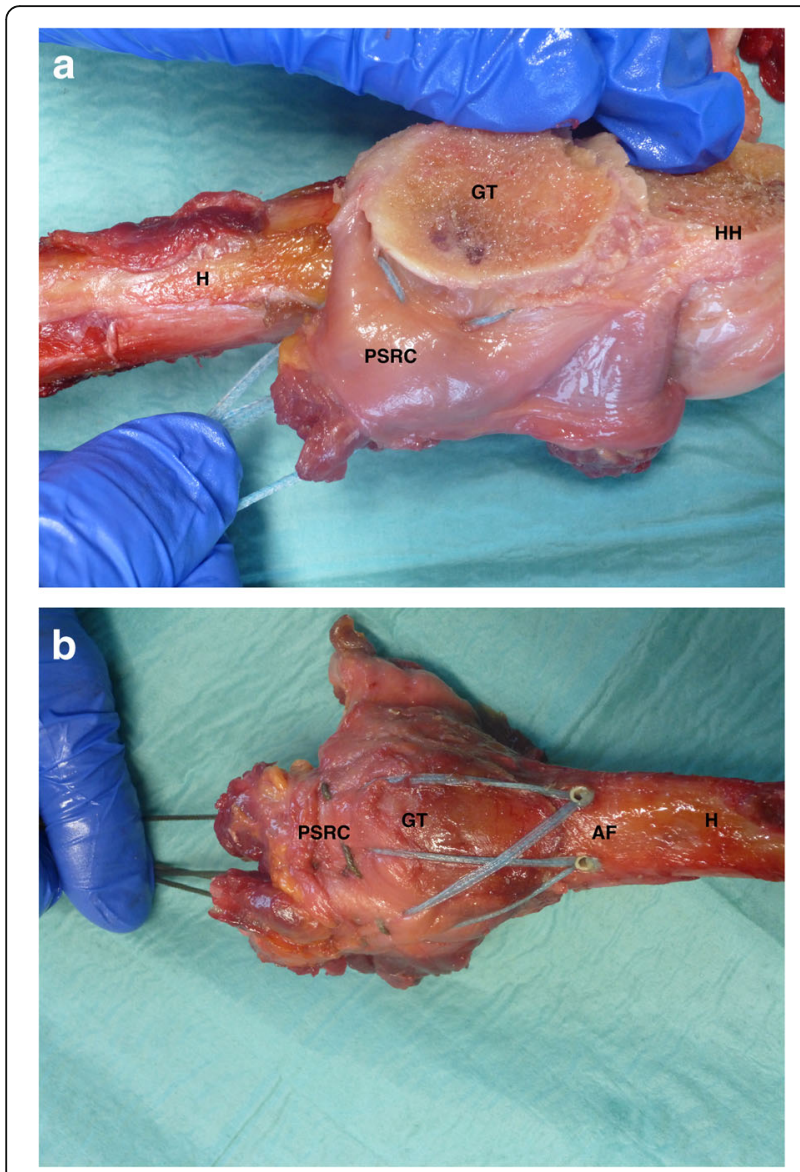

Fig. 2 a-c: Single Row Fixation; (H: Humerus; HH: Humeral Head; GT: Greater Tuberosity; PSRC: Postero-superior Rotator Cuff; AF: Anchor Fixation) with an accuracy of $.025 \mathrm{~mm}$. The specimens were then fixed to an electrodynamic material testing machine (E3000 with Instron Dynacell -Measuring range +/-3Kn-; Instron Ltd., High Wycombe, United Kingdom)(Fig. 4).

During mechanical tests, the humeral shaft was fixed at $0^{\circ}$ of abduction. To simulate abduction a braided, abrasion-resistant wire (Black Cat Power Leader Rhino, Tostedt, Germany) was used to reinforce the SSP and ISP tendons. The ends of the wire were fixed to the mechanical testing machine to apply an axial tensile load. The force was distributed equally between SSP and ISP tendons (50:50). The muscle tendons were preloaded with $50 \mathrm{~N}$. Starting with $50 \mathrm{~N}$ load level for cyclic loading the force was increased by $40 \mathrm{~N}$ every 1000 cycles until failure.

Ultimate failure load $[\mathrm{N}]$, along with displacement [1 $\mathrm{mm}]$, and mode of failure were recorded for all specimens. Failure was defined as fracture of the humeral neck, anchor loosening, suture rupture or complete dislocation of the greater tuberosity.

\section{Statistical analysis}

A post hoc power analysis using G*Power (version 3.1.9.2; Franz Paul, Kiel, Germany) was performed to determine the power of the study. On the basis of the results of the Fisher exact test, an effect size of 0.85 was calculated. With this effect size, an a of .05 , and the sample size of 6 , a power of 0.80 was calculated. For statistical analysis, SPSS software (version 22.0; IBM, New York, NY) was used. Normal distribution was tested and confirmed with the Kolmogorov- Smirnov test. Quantitative parameters are given as means, standard deviations, and 95\% confidence intervals. To evaluate the differences regarding BMD, specimen age, and load to failure between groups, a 1-way analysis of variance with
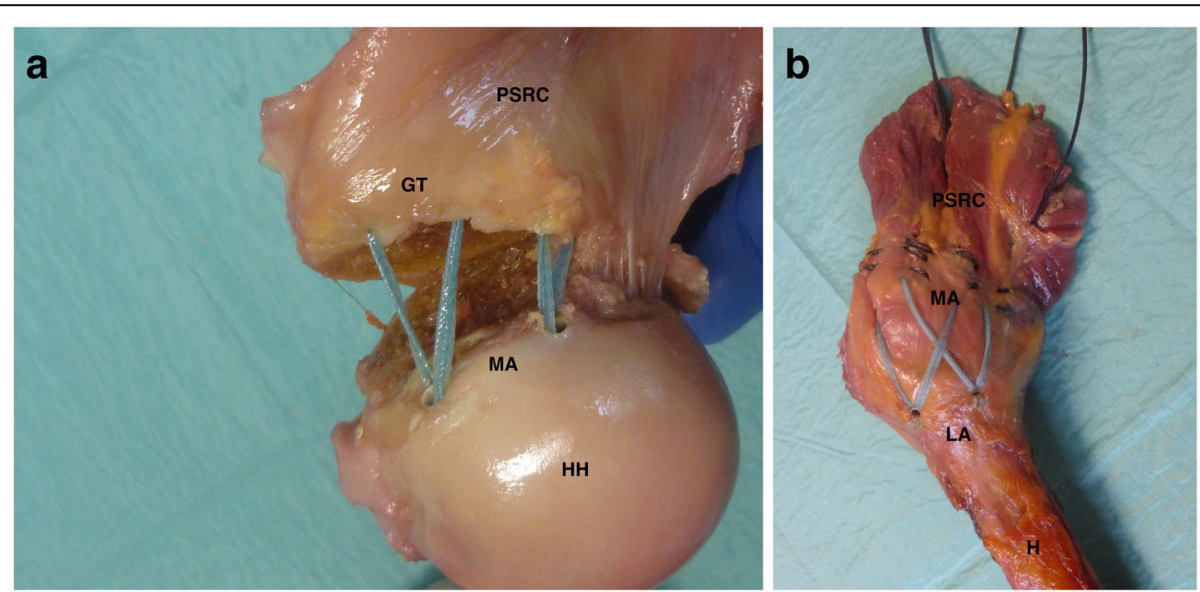

Fig. 3 a-b: Double Row Fixation; (H: Humerus; HH: Humeral Head; GT: Greater Tuberosity; PSRC: Postero-superior Rotator Cuff; MA: Medial Anchors; LA: Lateral Anchors) 


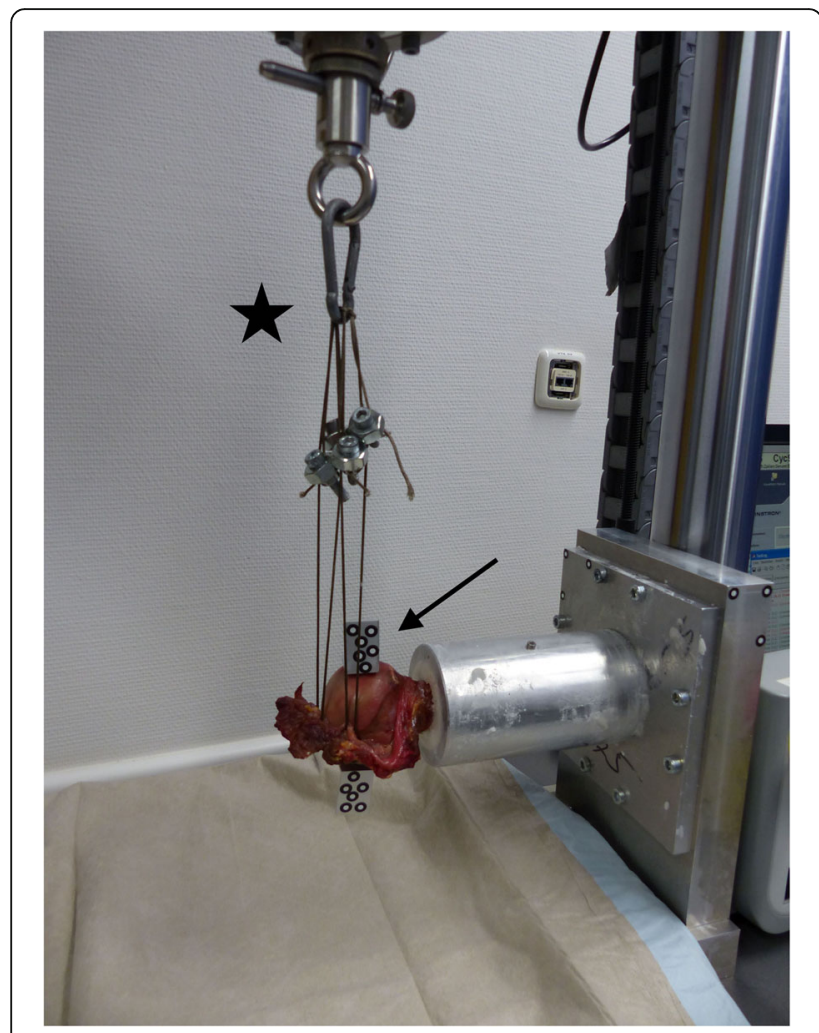

Fig. 4 Mechanical Testing Setup

a Tukey post hoc test was used. The Fisher exact test was used to analyze the failure modes between the testing groups. A significance level of $p<.05$ was accepted as a statistically significant difference.

\section{Results}

There were no significant differences between groups concerning BMD, morphology, and age (n.s.).

Regarding the mean cyclic load to failure (LtF) of suture anchor reconstruction in standardized fractures of the greater tuberosity, there were higher values in the DR group (649 $\mathrm{N} ; \pm 176$ ) compared to the SR group (490 N; \pm 145). (Fig. 5).

However, no significant difference could be seen between groups $(p=.12)$.

In the SR group the mean number of cycles to failure were $10,782 \pm 4203$ whereas construct failure could be observed in the DR group at $14317 \pm 4450$ cycles in average.

During the testing it was recognized that a $5 \mathrm{~mm}$ displacement - as defined in prior studies $[3,4,9,17,25]$ could only be achieved in one fourth of all specimen before construct failure. Thus, it was preferred to measure the applied force $(\mathrm{N})$ at $1 \mathrm{~mm}$ displacement to receive objective and comparable results between the groups. In the SR group the mean applied load for $1 \mathrm{~mm}$ displacement was $276.7 \mathrm{~N} \pm 46.2$ whereas in the DR group an

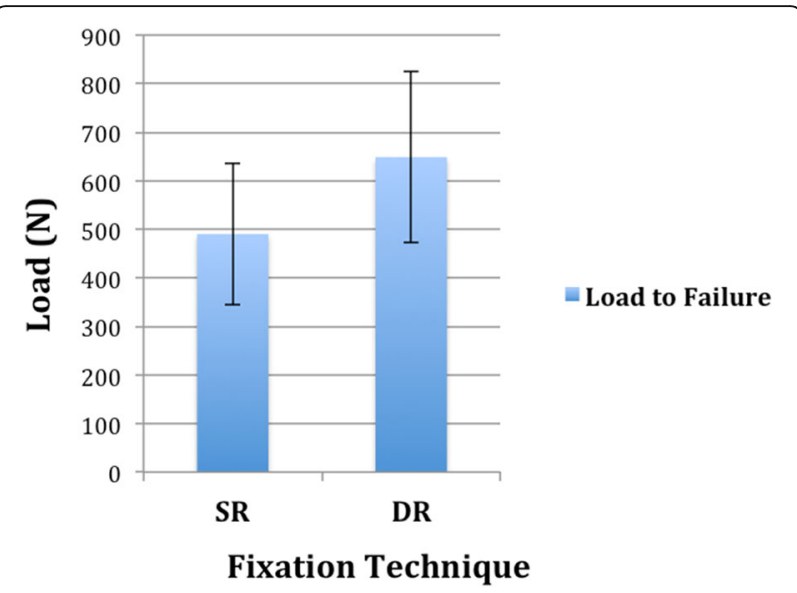

Fig. 5 Load to failure: Comparison of ultimate failure loads for single and double row fixation of greater tuberosity fractures $(p=.12)$. Data are presented as mean and standard deviation $(\mathrm{N})$

average load of $259.5 \mathrm{~N} \pm 61.8$ could be observed to achieve $1 \mathrm{~mm}$ displacement.

However, there was no difference regarding the loads for $1 \mathrm{~mm}$ displacement between single and double row fixation of greater tuberosity fractures $(p=.65)$ (Fig. 6).

\section{Mode of failure}

In the SR group the constructs failed because of fracture at least $2 \mathrm{~cm}$ distal to the humeral surgical neck in three cases (50\%) whereas a pull-out of the anterior anchor of the greater tuberosity could be seen in one specimen $(16,6 \%)$. In the DR group three specimens (50\%) failed due to fracture at least $2 \mathrm{~cm}$ distal to the humeral surgical neck. A pull-out of the anterior medial anchor of the greater tuberosity could also be seen in one specimen (16,6\%). A rupture of the abrasion-resistant wire fixation occurred in two specimens $(33,3 \%)$ at $\geq 650 \mathrm{~N}$ in the SR and the DR group,

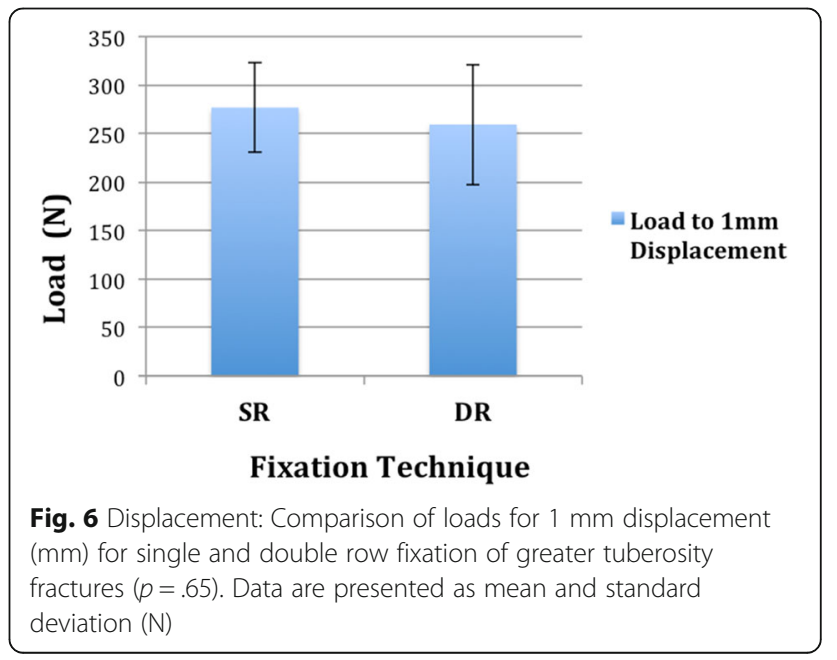


respectively. Therefore, there was no evident difference regarding failure modes between groups.

\section{Discussion}

In this biomechanical in-vitro study of arthroscopic suture anchor fixation techniques for greater tuberosity fractures, there was no significant difference between single-row and double-row repair regarding load to failure and applied traction force for $1 \mathrm{~mm}$ fracture displacement. Thus the studies primary hypothesis must be rejected. To the best of our knowledge, this is the first study presenting biomechanical data on single- vs. double-row knotless suture anchor repair of greater tuberosity fractures.

Treatment modalities of greater tuberosity fractures include conservative treatment, open, minimally invasive percutaneous, and arthroscopic procedures $[9,11,13$, $16,17,24,26,27]$. It has been shown that nonoperative management reveals disappointing results in case of displaced fractures of the greater tuberosity [26]. Thus surgical treatment is advised if superior displacement of $\geq 3$ $5 \mathrm{~mm}$ occurs to avoid malunion of the fractured greater tuberosity.

During the last years the focus has moved towards arthroscopic techniques $[6,12,14,16,23,24]$ due to the benefit of reduced pain, soft tissue damage and skin incision. Additionally arthroscopy allows to inspect the entire glenohumeral joint as well as to address concomitant pathologies in one session [20]. Besides, arthroscopic suture anchor repair using knotless implants may reduce operative time, simplifies the procedures and waives suture knots. One of the benefits of suture fixation is that it depends on the strength of the rotator cuff tendons themselves and not only on the bone quality.

Furthermore, this technique seems to be superior to other rigid methods like screws regarding load to failure and load to $5 \mathrm{~mm}$ displacement [17]. This represents an important recognition as fractures of the greater tuberosity are commonly comminuted and usually seen in young and active patients [1, 18]. Furthermore, in case of comminuted fragments of the greater tuberosity or in older patients with osteopenic bone [10] a wider contact surface of refixation could be helpful regarding healing and stability [30]. In addition, no additive surgery to remove osteosynthesis material is needed.

A gradual increase of greater tuberosity displacement that requires intervention - usually described as $3-5 \mathrm{~mm}$ displacement $[3,4,9,17,25]$ - could not be seen in this study.

Neither in the single-row nor in the double-row group all specimens consequently reached these two greater tuberosity displacement landmarks before construct failure.

This could be explained by an increased stability of the fixation method compared to rigid systems like two cancellous screws and due to the fixation of the entire tendinous insertion of the postero-superior rotator cuff (SSP/ISP). To receive objective and comparable results between groups, the applied traction force $(\mathrm{N})$ was analyzed at $1 \mathrm{~mm}$ displacement (277 N (SR) vs. $260 \mathrm{~N}$ (DR)). Consequently, no fixation technique (SR vs. DR) showed significant advantages over the other. In this study medial row of the DR technique was not tied as knotless suture tape systems were used. This may have affected the $1 \mathrm{~mm}$ displacement that occurred more easily in the double row repair than the single row repair.

In their biomechanical study Lin et al. [17] described a 3 and $5 \mathrm{~mm}$ displacement of the greater tuberosity in the double-row suture anchor group at $262.5 \mathrm{~N}$ and $370.3 \mathrm{~N}$ load as well as in the suture-bridge technique at $321 \mathrm{~N}$ and $398.5 \mathrm{~N}$ load. In the two-screw fixation group a 3 and $5 \mathrm{~mm}$ displacement was apparent at a load of $186.7 \mathrm{~N}$ and $249.2 \mathrm{~N}$, respectively.

This shows that the applied traction forces for $1 \mathrm{~mm}$ displacement are similar to the loads described by Lin et al. [17] for $3 \mathrm{~mm}$ displacement.

The ultimate failure loads in the study of Lin et al. [17] were $480 \mathrm{~N}$ (Double-row), $493.3 \mathrm{~N}$ (Suture-bridge) and $340 \mathrm{~N}$ for the two-screw fixation. The present study showed comparable failure loads in the SR group $(490 \mathrm{~N})$ and even higher loads in the DR group (649 N).Regarding failure modes, a fracture of the humeral surgical neck was noted in $50 \%$ of both groups indicating that the construct itself was stronger than the native bone. Lin et al. [17] also described a fracture of the humeral surgical neck as common failure mode. They also found a dislocation of the anterior anchor in one specimen of the Suture Bridge group.

In the present study, dislocation of a suture anchor was noticed in only one specimen (16,6\%) per group.

A rupture of the abrasion-resistant wire fixation occurred twice $(33,3 \%)$ in each group at more than $650 \mathrm{~N}$ in our study. This high load of failure underlines the findings of very stabile fixation methods - single- and double row. However, both fixation constructs are expected to tolerate significantly higher loads than the maximal supraspinatus force of $\sim 302 \mathrm{~N}$ [5]. This might be explained by the fixation method. In this study the common footprint of the postero-superior rotator cuff in line with the SSP/ISP force vector was fixed instead of the sole supraspinatus tendon as formerly described $[3,17]$. This was performed due to the described interdigitation of infraspinatus (ISP) and supraspinatus (SSP) fibres [7] with overlapping of the fibres [21] at the humeral footprint. Furthermore an extend of the infraspinatus' footprint along most of the highest facet of the greater tuberosity [22] is reported [19].

In addition FibreTape instead of FibreWire was used for suture anchor material that could also be responsible 
for the different failure loads although Ishak et al. [11] reported on equivalent load capacity. Nevertheless single SR reconstruction is an important mode of fixation. However, it may result in over reduction of the bone fragment distally. The major advantage of the double row technique is that the bone fragment is fixed at the osteoarticular junction and avoids over reduction of the fragment.

In clinical practice significantly softer bone stock is sometimes seen medially to the fracture. If there are concerns regarding the stability of the medial row of the double row reconstruction, single row fixation represents a high quality alternative but with biomechanically reduced load to failure. One of the major advantages of this suture technique is that fixation depends on the strongest tissue in the area which is the rotator cuff tendon itself.

Besides, in case of comminuted fractures of the greater tuberosity or in osteopenic bone, this study could provide important information regarding suture anchor reconstruction that underlines the clinical relevance of our study.

\section{Limitations}

There are several limitations to this study that need to be considered. First, using an in-vitro cadaveric model, the study can only assess direct postoperative (time-zero) stability, not accounting for any biological influences during the healing period.

In addition, the influence of abduction, elevation or rotation and its shear, compression, or torsion forces to the fixation construct were not assessed what may affect the clinical situation. However, used postero-lateral force of SSP and ISP is closer to clinically reality than previous setups.

Also, the artificially induced fractures of the greater tuberosity may not reflect completely the conditions in vivo as fractures of the greater tuberosity are often comminuted. However, comminuted fractures are difficult to induce and reproduce artificially.

In addition, the influence of poor bone quality on the fixation stability was not assessed. Furthermore, although sample size is comparable to existing studies $[3,17]$ focussing on this topic, the study may be underpowered.

\section{Conclusion}

In this biomechanical study arthroscopic single- and double-row suture anchor repair of isolated greater tuberosity fractures seem to be viable options for treatment. Therefore, in the setting of reduced bone quality and weak anchorage of the medial row anchors, the authors perform a single row repair in daily clinical practice.

\section{Abbreviations}

AF: Anchor fixation; DR: Double row; GT: Greater Tuberosity; H: Humerus; HH: Humeral Head; ISP: Infraspinatus; LA: Lateral anchors; MA: Medial anchors; PSRC: Postero-superior rotator cuff; SR: Single Row; SSP: Supraspinatus

\section{Acknowledgments}

This study was conducted at the Institute of Biomechanics (PMU Salzburg), Berufsgenossenschaftliche Unfallklinik Murnau, Murnau, Germany. This work was supported by the German Research Foundation (DFG) and the Technical University of Munich (TUM) in the framework of the Open Access Publishing Program.

\section{Funding}

No source of outside financial funding was used for this study. Arthrex Inc. provided the specimen and implants but was not involved in study design, implementation, data collection, analysis, and/or interpretation.

\section{Availability of data and materials}

All relevant data supporting the conclusions are included within the article and tables.

The raw data are part of a greater institutional investigation project. The datasets used and/or analyzed during the current study are available from the corresponding author on reasonable request.

\section{Authors' contributions}

GS: Study design, data analysis \& interpretation, abstract, introduction, material \& methods, results, and discussion. TS: Study design, data acquisition/analysis, and results. FM: Data analysis, statistical analysis, results, and discussion. JEP: Data collection, data analysis, and critical review. AG-V: Study design, figures, introduction, and critical review. JH: Concept/realization biomechanical testing setup system, data acquisition, and results. MW: Concept/realization biomechanical testing setup system, data acquisition, and methods. PA: Study design, data interpretation, and critical review. ABI: Study conception and critical review. SB: Study conception, data interpretation, and critical review. All authors agreed to be accountable for all aspects of the work and approved the final manuscript to be published.

Ethics approval and consent to participate

The study was approved by the Ethics Committee of the Technical University Munich, Munich, Germany (Vote-Nr: 382/15).

Cadavers were provided by the Institute of Forensic Medicine, Ludwig

Maximilians University Munich. Written consent was obtained from next of kin.

Consent for publication

Not applicable.

\section{Competing interests}

AB Imhoff and F Martetschläger are consultants for Arthrex Inc. The other authors declare, that they have no competing interests.

Tim Saier is a member of the Editorial Board of BMC Musculoskeletal Disorders.

\section{Publisher's Note}

Springer Nature remains neutral with regard to jurisdictional claims in published maps and institutional affiliations.

\section{Author details}

${ }^{1}$ Department of Orthopedic Sports Medicine, Klinikum rechts der Isar, Technical University Munich, Ismaninger Str. 22, 81675 Munich, Germany. ${ }^{2}$ Department of Orthopedics and Trauma Surgery, Krankenhaus Barmherzige Brüder, Munich, Germany. ${ }^{3}$ Department of Reconstructive Joint Surgery and Sportstraumatology, Berufsgenossenschaftliche Unfallklinik Murnau, Murnau, Germany. ${ }^{4}$ Center for Shoulder and Elbow Surgery, ATOS Clinic Munich, Munich, Germany. ${ }^{5}$ Department of Trauma Surgery, Klinikum Augsburg, Augsburg, Germany. ${ }^{6}$ Institute of Biomechanics, Berufsgenossenschaftliche Unfallklinik Murnau, Murnau, Germany. ${ }^{7}$ Institute of Biomechanics, Paracelsus Medical University, Salzburg, Austria. ${ }^{8}$ Orthopädisches Fachzentrum, Weilheim, Germany.

Received: 30 November 2016 Accepted: 20 November 2017 Published online: 01 December 2017

\section{References}

1. Bahrs $C$, Lingenfelter E, Fischer F, Walters EM, Schnabel M. Mechanism of injury and morphology of the greater tuberosity fracture. Journal of shoulder and 
elbow surgery/American Shoulder and Elbow Surgeons [et al.]. 2006;15:140-7. https://doi.org/10.1016/j.jse.2005.07.004

2. Bono CM, Renard R, Levine RG, Levy AS. Effect of displacement of fractures of the greater tuberosity on the mechanics of the shoulder. The Journal of bone and joint surgery British volume. 2001;83:1056-62.

3. Braunstein V, Wiedemann E, Plitz W, Muensterer OJ, Mutschler W, Hinterwimmer S. Operative treatment of greater tuberosity fractures of the humerus-a biomechanical analysis. Clin Biomech. 2007;22:652-7. https://doi. org/10.1016/j.clinbiomech.2007.03.002.

4. Brunner UH. Humeruskopffraktur. In: Habermayer P, editor. Schulterchirurgie. Urban und Fischer, München, pp; 2002. p. 404-29.

5. Burkhart SS. A stepwise approach to arthroscopic rotator cuff repair based on biomechanical principles. Arthroscopy : the journal of arthroscopic \& related surgery : official publication of the Arthroscopy Association of North America and the International Arthroscopy Association. 2000;16:82-90.

6. Cadet ER, Ahmad CS (2007) Arthroscopic reduction and suture anchor fixation for a displaced greater tuberosity fracture: a case report. Journal of shoulder and elbow surgery/American Shoulder and Elbow Surgeons [et al.] 16:e6-e9. doi:https://doi.org/10.1016/j.jse.2006.08.010.

7. Clark JM, Harryman DT, 2nd (1992) Tendons, ligaments, and capsule of the rotator cuff. Gross and microscopic anatomy. The Journal of bone and joint surgery American volume 74:713-725.

8. Flatow EL, Cuomo F, Maday MG, Miller SR, Mcllveen SJ, Bigliani LU. Open reduction and internal fixation of two-part displaced fractures of the greater tuberosity of the proximal part of the humerus. The Journal of bone and joint surgery American. 1991;73:1213-8.

9. Gerber C, Warner JJP (1997) Alternatives to hemiarthroplasty for complex proximal-humerus fractures. In: Warner JJP, lannotti JP, Gerber C (eds) Complex and revision problems in shoulder surgery. Lippincott-raven, Philadelphia, pp pp. 215-243.

10. Green A, Izzi J Jr. Isolated fractures of the greater tuberosity of the proximal humerus. Journal of shoulder and elbow surgery/American Shoulder and Elbow Surgeons [et al]. 2003;12:641-9. https://doi.org/10.1016/ S1058274602868112.

11. Ishak C, Sahajpal D, Chiang A, Atallah W, Kummer F, Jazrawi LM. Fixation of greater tuberosity fractures: a biomechanical comparison of three techniques. Bulletin. 2006;63:98-9.

12. Ji JH, Shafi M, Song IS, Kim YY, McFarland EG, Moon CY. Arthroscopic fixation technique for comminuted, displaced greater tuberosity fracture. Arthroscopy : the journal of arthroscopic \& related surgery : official publication of the Arthroscopy Association of North America and the International Arthroscopy Association. 2010;26:600-9. https://doi.org/10. 1016/j.arthro.2009.09.011.

13. Keener JD, Parsons BO, Flatow EL, Rogers K, Williams GR, Galatz LM. Outcomes after percutaneous reduction and fixation of proximal humeral fractures. Journal of shoulder and elbow surgery/American Shoulder and Elbow Surgeons [et al.]. 2007;16:330-8. https://doi.org/10.1016/j.jse.2006.09.006.

14. Kim YS, Lee BG, Rhee YG, Lee CH. Arthroscopic reduction and internal fixation for displaced anterior glenoid rim and greater tuberosity fractures. Journal of orthopaedic science : official journal of the Japanese Orthopaedic Association. 2014;19:497-501. https://doi.org/10.1007/s00776-012-0309-6.

15. Kristiansen $B$, Angermann $P$, Larsen TK. Functional results following fractures of the proximal humerus. A controlled clinical study comparing two periods of immobilization. Arch Orthop Trauma Surg. 1989;108:339-41.

16. Lee SU, Jeong C, Park IJ. Arthroscopic fixation of displaced greater tuberosity fracture of the proximal humerus. Knee surgery, sports traumatology, arthroscopy : official journal of the ESSKA. 2012;20:378-80. https://doi.org/10.1007/s00167-011-1748-x.

17. Lin CL, Hong CK, Jou IM, Lin CJ, FC S, WR S. Suture anchor versus screw fixation for greater tuberosity fractures of the humerus-a biomechanical study. Journal of orthopaedic research: official publication of the Orthopaedic Research Society. 2012;30:423-8. https://doi.org/10.1002/jor.21530.

18. Lind $T$, Kroner $K$, Jensen J. The epidemiology of fractures of the proximal humerus. Arch Orthop Trauma Surg. 1989;108:285-7.

19. Lumsdaine W, Smith A, Walker RG, Benz D, Mohammed KD, Stewart F. Morphology of the humeral insertion of the supraspinatus and infraspinatus tendons: application to rotator cuff repair. Clin Anat. 2015;28:767-73. https:// doi.org/10.1002/ca.22548

20. Maman E, Dolkart O, Chechik O, Amar E, Rak O, Rath E, Mozes G. Arthroscopic findings of coexisting lesions with greater tuberosity fractures. Orthopedics. 2014;37:e272-7. https:/doi.org/10.3928/01477447-20140225-60.
21. Minagawa H, Itoi E, Konno N, Kido T, Sano A, Urayama M, Sato K. Humeral attachment of the supraspinatus and infraspinatus tendons: an anatomic study. Arthroscopy : the journal of arthroscopic \& related surgery: official publication of the Arthroscopy Association of North America and the International Arthroscopy Association. 1998;14:302-6.

22. Mochizuki T, Sugaya H, Uomizu M, Maeda K, Matsuki K, Sekiya I, Muneta T, Akita K. Humeral insertion of the supraspinatus and infraspinatus. New anatomical findings regarding the footprint of the rotator cuff. The Journal of bone and joint surgery American. 2008;90:962-9. https://doi.org/10.2106/ JBJS.G.00427.

23. Park SE, Jeong JJ, Panchal K, Lee JY, Min HK, Ji JH. Arthroscopic-assisted plate fixation for displaced large-sized comminuted greater tuberosity fractures of proximal humerus: a novel surgical technique. Knee surgery, sports traumatology, arthroscopy: official journal of the ESSKA. 2015; https:// doi.org/10.1007/s00167-015-3805-3.

24. Park SE, Ji JH, Shafi M, Jung JJ, Gil HJ, Lee HH. Arthroscopic management of occult greater tuberosity fracture of the shoulder. European journal of orthopaedic surgery \& traumatology: orthopedie traumatologie. 2014;24: 475-82. https://doi.org/10.1007/s00590-013-1212-3.

25. Park TS, Choi IY, Kim YH, Park MR, Shon JH, Kim SI. A new suggestion for the treatment of minimally displaced fractures of the greater tuberosity of the proximal humerus. Bulletin. 1997;56:171-6

26. Platzer $P$, Thalhammer $G$, Oberleitner $G$, Kutscha-Lissberg F, Wieland T, Vecsei V, Gaebler C. Displaced fractures of the greater tuberosity: a comparison of operative and nonoperative treatment. J Trauma. 2008;65: 843-8. https://doi.org/10.1097/01.ta.0000233710.42698.3f.

27. Resch $H$, Hubner C, Schwaiger R. Minimally invasive reduction and osteosynthesis of articular fractures of the humeral head. Injury. 2001; 32(Suppl 1):SA25-32

28. Scheibel M, Martinek V, Imhoff AB. Arthroscopic reconstruction of an isolated avulsion fracture of the lesser tuberosity. Arthroscopy : the journal of arthroscopic \& related surgery: official publication of the Arthroscopy Association of North America and the International Arthroscopy Association. 2005;21:487-94. https://doi.org/10.1016/j.arthro.2004.11.012.

29. Taverna E, Sansone V, Battistella F. Arthroscopic treatment for greater tuberosity fractures: rationale and surgical technique. Arthroscopy : the journal of arthroscopic \& related surgery: official publication of the Arthroscopy Association of North America and the International Arthroscopy Association. 2004;20:e53-7.

30. Tuoheti Y, Itoi E, Yamamoto N, Seki N, Abe H, Minagawa H, Okada K, Shimada Y. Contact area, contact pressure, and pressure patterns of the tendon-bone interface after rotator cuff repair. Am J Sports Med. 2005;33: 1869-74. https://doi.org/10.1177/0363546505278256.

31. Williams GR Jr, Wong KL. Two-part and three-part fractures: open reduction and internal fixation versus closed reduction and percutaneous pinning. The Orthopedic clinics of North America. 2000;31:1-21.

\section{Submit your next manuscript to BioMed Central and we will help you at every step:}

- We accept pre-submission inquiries

- Our selector tool helps you to find the most relevant journal

- We provide round the clock customer support

- Convenient online submission

- Thorough peer review

- Inclusion in PubMed and all major indexing services

- Maximum visibility for your research

Submit your manuscript at www.biomedcentral.com/submit
C) Biomed Central 